\title{
Isothermal micro-channel gas flow using a hydrodynamic model with dissipative mass flux
}

\author{
Nishanth Dongari, S Kokou Dadzie, Yonghao Zhang and Jason M Reese \\ Department of Mechanical Engineering, University of Strathclyde, Glasgow G1 1XJ, Scotland, United Kingdom \\ nishanth.dongari@strath.ac.uk
}

\begin{abstract}
In this paper we investigate the problem of isothermal pressure driven gas flow through a channel using a continuum equations set with a mass diffusion correction to the mass-density equation. The additional term is invoked as a way of releasing the local-equilibrium assumption in the presence of strong local gradients. Then the dissipative mass flux is proportional to local density/pressure gradient which is constant in a cross section and results in a correction to the velocity profile. Subsequently, better mass-flow rate predictions are obtained than those obtained using Maxwell-type first order slip boundary conditions. Results indicate that the mass diffusion correction captures the "Knudsen paradox" and change in curvature of the streamwise pressure profile without the need for setting higher order slip boundary conditions.
\end{abstract}

Keywords: mass diffusion; microchannel gas flow; Knudsen paradox; pressure distribution; slip flows

PACS: 47.61.-k, 47.61.Cb, 47.61.Fg

\section{INTRODUCTION}

Technological advancements in Micro-Electro-Mechanical Systems (MEMS) have rekindled interest in fundamental scientific investigations concerning physical phenomena such as boundary slip of gas flows at small length scales [1]. In microscale rarefied gas flows, deviations from local thermodynamic equilibrium are significant, and the extent of this deviation can be quantified in terms of the Knudsen number $(K n)$, which is the ratio of the gas molecular mean free path $(\lambda)$ to the characteristic system length scale $(H)$. Experimental investigations [1] have confirmed that the conventional Navier-Stokes-Fourier (NSF) equations, in their traditional form with no slip boundary condition, underpredict experimental mass flow rates in microscale gas flows, and deviations become more significant with increase in Knudsen number. The phenomenon of enhanced mass flow rates is typically addressed by applying the Maxwell slip boundary condition in conjunction with the continuum equations. However, Maxwell-type first order slip models are accurate only up to small values of the $K n$, and also fail to predict the well established Knudsen paradox phenomenon [2].

Higher order slip boundary conditions have been proposed to stretch the applicability of continuum models over a larger range of Knudsen numbers, but, they have been typically closed with slip coefficients that may themselves vary with Knudsen number. Behaviour of isothermal rarefied gases can be described by the Boltzmann equation [3], and the mass flow rate over the full range of Knudsen number predicted [4]. However, solution methods require an assumption on the local pressure that does not allow an investigation of the actual pressure profile $[3,4]$.

Recently, it has been suggested by several researchers [5-9] that the constitutive assumption in-built with the standard forms of the Navier-Stokes-Fourier (NSF) equations need to be extended to render them applicable for fluid flows with strong local gradients of density and temperature. Dadzie et al. [9] proposed a microscopic volume-based kinetic approach analyzing the molecular spatial distribution; this led to a set of continuum equations incorporating a volume diffusive flux, which also behaves like a mass diffusion flux. These kind of extended continuum set of equations improve predictions of certain flows in the finite Knudsen number regime [10, 11].

In this paper, we assess the implication of a mass diffusion correction for isothermal gas flow along a micro channel. We show that it can predict the actual pressure distributions in these flows. Axial pressure profiles at various pressure ratios are validated with experimental data, and the effects of Knudsen number on the curvature of the pressure profiles is analyzed. Further results indicate that the mass diffusion correction captures the "Knudsen paradox" (i.e. the minimum in flow rate) without the need to set higher-order slip boundary conditions. Moreover, our mass diffusion model offers a more reliable explanation of the Knudsen paradox. 


\section{A SIMPLIFIED MASS DIFFUSION CONTINUUM MODEL}

As our objective is to analyse the implication of a dissipative mass correction, we consider a continuum model consisting of the classical conservative equations of mass and momentum, modified by a dissipative mass-density flux, and written as follow:

Mass-density

$$
\frac{\partial \rho}{\partial t}+\nabla \cdot\left[\rho U+\mathbf{J}_{\mathbf{m}}\right]=0
$$

\section{Momentum}

$$
\frac{\partial \rho U}{\partial t}+\nabla \cdot[\rho U U]+\nabla \cdot[p \mathbf{I}+\Pi]=0
$$

where quantities $\mathbf{J}_{\mathbf{m}}$ and $\Pi$ are given a Fick's law diffusive flux representation as:

$$
\begin{gathered}
\Pi=-v\left[\nabla(\rho U)+(\nabla(\rho U))^{t r}\right]+\eta \nabla \cdot(\rho U) \mathbf{I}, \\
\mathbf{J}_{\mathbf{m}}=-\kappa_{m} \nabla \rho .
\end{gathered}
$$

The energy equation is omitted in the above set of equations, as the considered flow problem is isothermal. In these equations, $\rho$ is the mass-density and $U$ is the flow velocity. Pressure $p$ and temperature $T$ are related by the equation of state: $p=\rho \Re T$, with $\Re$ being the specific gas constant and $\mathbf{I}$ is the identity matrix. Quantities $\mathbf{J}_{\mathbf{m}}$ and $\Pi$ denote diffusive fluxes, in addition to the convective transport fluxes, for mass and momentum respectively. These diffusive terms are all modeled here for simplicity by a Fick's law type of expression. Compared with traditional expressions of the continuum conservation equations, it is to note that an additional diffusive term, $\mathbf{J}_{\mathbf{m}}$, introduced in the massdensity equation marks the difference. A non-vanishing $\mathbf{J}_{\mathbf{m}}$ may find an interpretation where local gradients are no longer negligible, or as a result of fluctuations in molecular spatial distributions [9]. The momentum density diffusivity coefficient (or kinematic viscosity) is $v$, that in turn defines a dynamic viscosity $\mu=\rho \nu$ and $\kappa_{m}$ is a mass diffusivity coefficient. In eq. (3), $\eta$ is the bulk viscosity so that $\eta=2 / 3 v$ corresponds to Stokes's assumption.

In a strict non-local-equilibrium derivation of the full set of macroscopic continuum equations, one may expect the additional non-equilibrium terms to effect momentum and energy balance. We next test the implication of the mass diffusion correction in the mass-density equation for isothermal pressure driven flow.

\section{ISOTHERMAL MICRO GAS FLOW}

We consider low speed isothermal gas flow along a microchannel in order to test the effect of our mass diffusion correction. The channel height $H$ is assumed to be much smaller than the channel width, so that the fluid essentially sees two infinite parallel plates, separated by a gap $H$, at coordinates $y= \pm H / 2$. The flow is assumed to be steady, two-dimensional and hydrodynamically developed so that any wall-normal velocity component may be neglected. The Schmidt number, $v / \kappa_{m}$, the ratio of the momentum diffusivity to mass diffusivity is assumed to be unity and hence $\kappa_{m}=v$.

Under the above assumptions, continuity and momentum Eqs. (1) and (2) reduce to the following form:

$$
\begin{gathered}
\frac{d \rho U}{d z}=\frac{d}{d z}\left(\frac{\mu}{\rho} \frac{d \rho}{d z}\right) \\
\frac{d \rho U U}{d z}=-\frac{d p}{d z}+v\left[\frac{4}{3} \frac{d^{2} \rho U}{d z^{2}}+\rho \frac{d^{2} U}{d y^{2}}\right]
\end{gathered}
$$

where $z$ and $y$ are streamwise and wall-normal coordinates, respectively. Although inertial and axial shear stress are retained in the above governing equations, they can be neglected if the ratio of the channel height to length is small $(H / L<<1)$. 

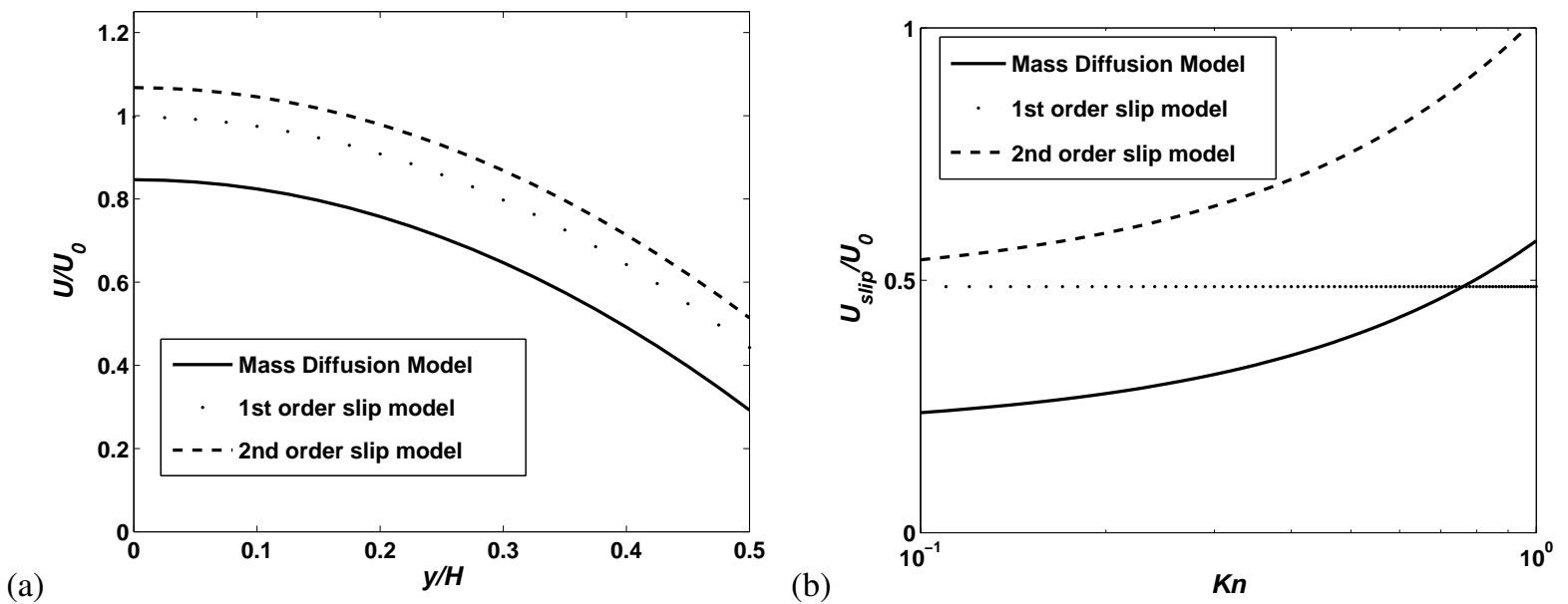

FIGURE 1. (a) Normalized cross-sectional velocity profile for micro-channel gas flows in the early transition flow regime $(K n=0.2)$, and comparison with slip models; (b) variation of normalized slip velocity as a function of Knudsen number, and comparison with slip models.

We require results for the flow velocity profiles across the channel, the pressure profile and some integral flow parameters, through solution of the governing equations (5)-(6) with the no-slip boundary condition $U=0$ at $y=$ $\pm H / 2$. Equations (5)-(6) can be solved numerically for given pressure values at the inlet and outlet of the channel. The wall-normal coordinate $y$ is normalized by $H$, and the axial velocity $U$ by the free-molecular velocity $U_{0}=$ $-2 H(\partial p / \partial z) /(\rho \sqrt{2 R T})[16]$.

\section{RESULTS AND DISCUSSION}

In the absence of any experimental data for the velocity profile, our present model results are compared with first and second-order slip flow models [15], acknowledging the notional analogies between Maxwell-type slip velocities and the diffusive velocity.

Figure 1(a) depicts the normalized axial velocity profile along the cross-stream direction for low speed gas flow at $K n=0.2$, which is just beyond the slip-flow regime. It is apparent that the mass difussion model systematically lies under both the slip solutions. Non-zero flow velocity at the wall is triggered in the present model through the additional mass diffusion correction, whereas traditionally slip effects are diffused through viscous stresses in the wall-adjacent layer in accordance with the slip-based boundary conditions prescribed at the walls.

In Figure 1(b), we present normalized slip velocities as a function of Knudsen number. First-order slip velocity turns out to be independent of Knudsen number, and is a higher value than the mass diffusion model for $K n<0.7$ and lower for $K n>0.7$. This constant value can also be qualitatively deduced by writing the first-order slip boundary condition in terms of the wall shear stress $\tau_{w}$, and expressing it in terms of pressure gradient using the momentum balance equation. Second-order slip is too high, and deviations become more significant with increasing Knudsen number. Although both slip models overpredict results for $K n<0.5$, they do not converge to the no-slip value in the continuum limit $(K n \rightarrow 0)$. Unlike the Maxwell-type first-order slip model, a mass diffusion correction imparts significant nonlinearities to the $K n$-dependence of the predicted slip velocity.

Knudsen's experimental work in the transition flow regime [2] revealed that there is a minimum in the normalized mass flow rate $G$ [4]. This is referred to as the Knudsen paradox in the literature, as the classical Navier-Stokes solution with no-slip boundary conditions fail to predict this phenomenon. In the standard Navier-Stokes solution, the mass flow rate is driven by the externally imposed pressure difference between the inlet and outlet of the channel. In the present model we have the additional diffusive mass flux, which is driven by local density gradients in the system due to imposed pressure gradient. Figure 2(a) shows the variation of $G$ with inverse Knudsen number, $\delta=\sqrt{\pi} /(2 K n)$. Present model results are validated against the experimental data from the continuum limit to the transition flow regime. For substantially low values of $K n$, i.e. in the limit of the continuum regime, the diffusive flux is negligibly small, so that the present model predictions are close to the no-slip solution, see Fig 2(b). However, as shown in 

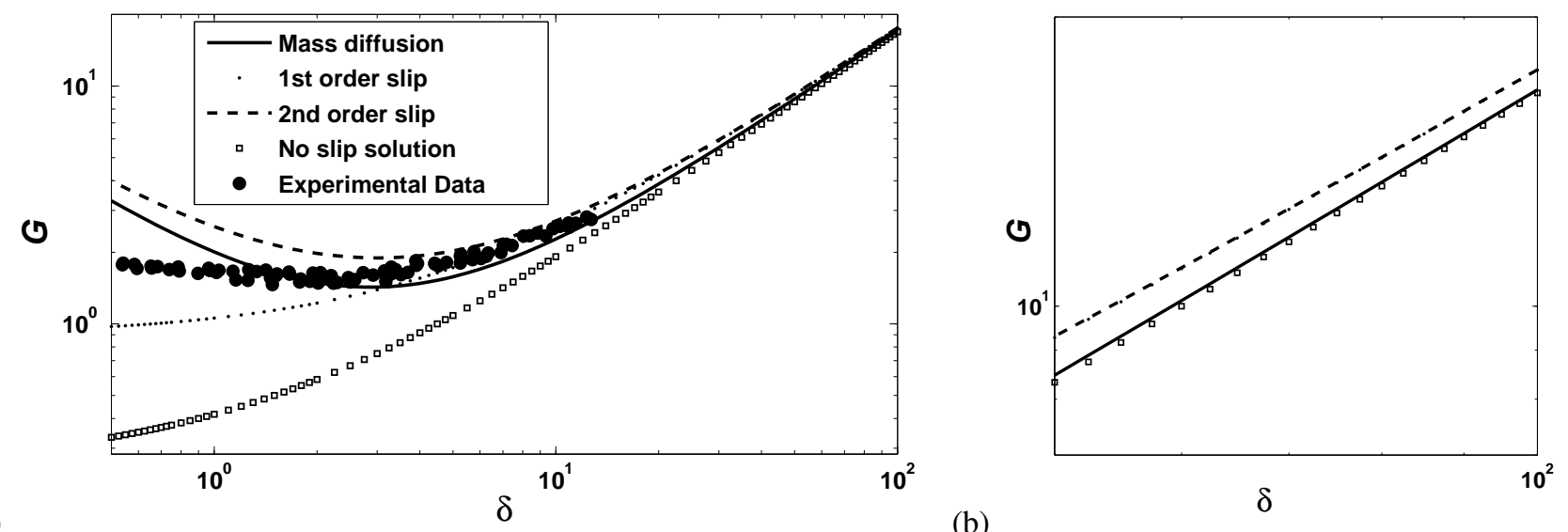

(a)

(b)

FIGURE 2. Variation of normalized mass flow rate as a function of inverse Knudsen number. Present model results are validated against the experimental data of Ewart et al. [4] and compared with no-slip, first- and second-order slip models.

Fig. 2(b), both the first and second order slip models do not converge to the no-slip solution in the continuum limit. Although this difference $\left(\left(G_{\text {slip }}-G_{n o-s l i p}\right) \sim 1\right)$ is almost negligible at high values of $G$, on physical grounds this may raise a fundamental question about current slip boundary conditions. With increasing Knudsen number, the additional diffusive flux becomes significant and deviates from the no-slip solution. Beyond a critical limit $(K n>0.3)$, the diffusive flux dominates the convective flux, which leads to the minimum (Knudsen paradox) in the flow rate profile. The diffusion model shows minor underpredictions compared with the experimental data in the early transition regime flow $(0.1<K n<0.25)$ and fair agreement up to $K n \sim 0.8$. First order slip model results are accurate up to $K n \sim 0.15$ but fail to predict the Knudsen paradox. The second order slip model shows good agreement with the measurements up to $K n \sim 0.4$, and predicts the Knudsen paradox.

Figure 3 presents a comparison of normalized streamwise pressure distributions against the experimental data of Pong et al. [13] at various pressure ratios (Pr). Here, pressure is normalized with the outlet pressure of the channel, and the pressure ratio is defined as the ratio of inlet to outlet pressures. The measurements by Pong et al. [13] were made by embedding measurement ports in a microchannel in which pressure transducers were mounted. The working gas was nitrogen and the outlet Knudsen number was 0.044 . The comparison between the present mass diffusion model and the data is good.

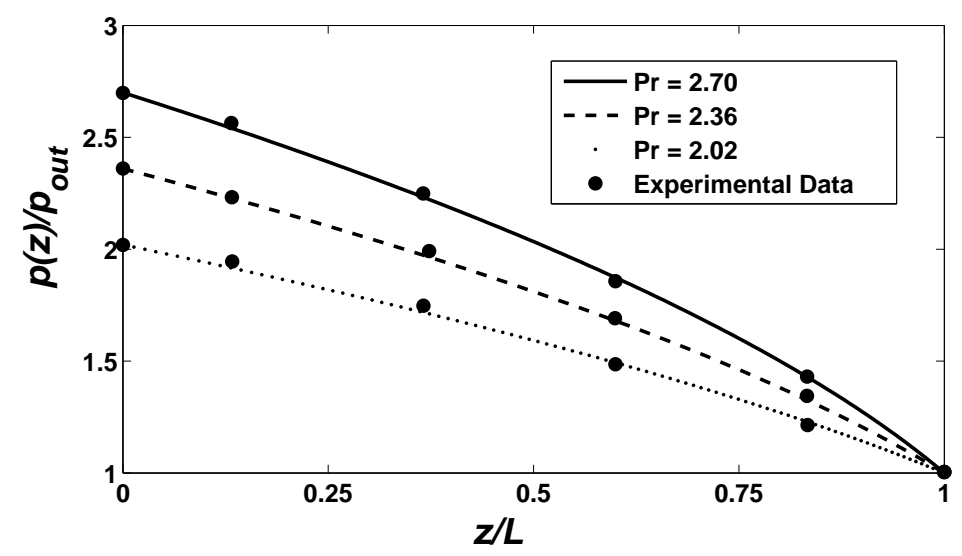

FIGURE 3. Comparison of pressure predicted by our simplified mass diffusion model against the experimental data of Pong et al. [13] for an outlet Knudsen number of 0.044 and various pressure ratios (Pr).

The diffusive mass effect on the streamwise pressure distribution can be assessed by varying the Knudsen number. Interesting results are observed as shown in the Fig. 4(a) for a pressure ratio of 3. The curvature of the pressure 


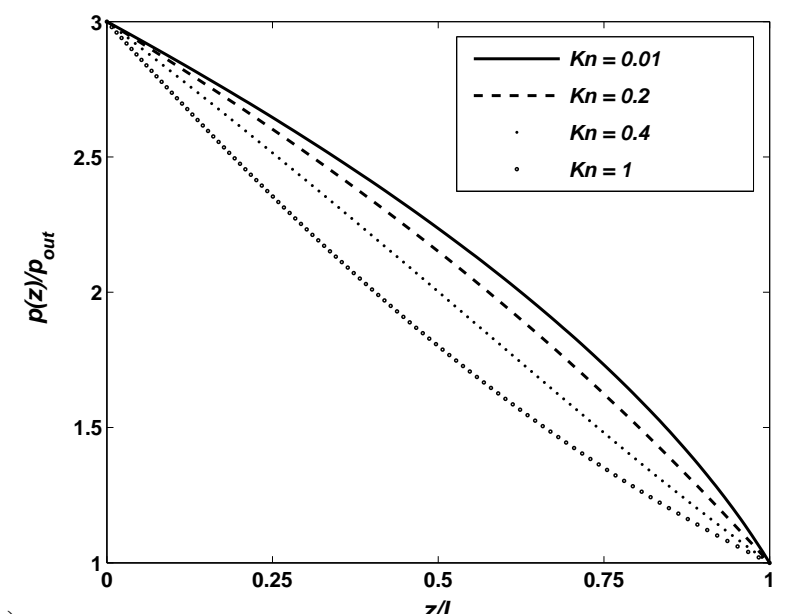

(a)

FIGURE 4. (a) Normalized pressure distribution along the streamwise direction for various Knudsen numbers, (b) dimensionless deviation of the pressure distribution from the linear pressure distribution as a function of the dimensionless distance.

variation decreases with an increase in Knudsen number, and the pressure distribution becomes linear in the early transition regime. With further increases in Knudsen number the curvature changes from convex (in the continuum and early transition regimes) to concave (in the late transition regime). Fig. 4(b) shows the dimensionless deviation of the streamwise pressure distribution from a linear pressure profile, as a function of dimensionless distance along the channel. With an increase in Knudsen number, the rarefaction effect tends to dominate compressibility effects, and the dimensionless deviation becomes zero at a Knudsen number of around 0.4. With further increases in Knudsen number, the dimensionless deviation changes from positive to negative value. In the slip flow regime, where compressibility effects are still dominant, the pressure profile is unsymmetrical with respect to the streamwise distance, but turns out to be quite symmetric in the transition regime at $K n=1$, where rarefaction/diffusive effects are dominant.

\section{CONCLUSIONS}

In this paper, theoretical studies of gas flows through micro-channel have been carried out from the continuum to the transition flow regime. Conventionally, isothermal microscale gas flow problems are studied either with the aid of Maxwell-type slip boundary conditions in conjunction with the continuum equations, or through the solution of the Boltzmann equation. We test a mass diffusion correction to the mass-density equation as an alternative model to the former approach. We find this addresses the enhanced mass flow rate phenomenon and the Knudsen paradox in the slip and transition flow regimes respectively. The diffusive mode of transport also introduces a slip-type of wall velocity, but in a somewhat natural way as the diffusive flux depends only on the axial pressure gradient, which is uniform across the channel cross-section. Effectively, this dissipative mass correction obviates the need to impose velocity slip boundary condition explicitly at the wall and predicts relatively more accurate results than the second-order slip model. Unlike Maxwell-type slip models, the mass diffusion solution asymptotes to the no-slip Navier-Stokes results in the continuum regime. It also allows the direct investigation of the non-linear pressure distribution, and predicts the change in curvature of the streamwise pressure profile with an increase in rarefaction (when the diffusive effects dominate the convective fluxes). Comparison with experimental mass flow rates suggest that this simple dissipative correction to the continuity equation improves the applicability of the continuum equations up to Knudsen numbers around 0.8 .

While it is important not to draw strong conclusions based on just one test case, this simple mass diffusion correction may motivate future work into understanding the origin of non-equilibrium physics in rarefied gas flows covering a wide range of Knudsen and Mach number. One may derive the dissipative mass flux model in terms of thermodynamic gradients through an appropriate kinetic equation, or through non-equilibrium thermodynamic considerations. In addition to a modification to the mass-density equation, local gradient-driven dissipative mass transport will also induce additional terms in the constitutive form of dissipative momentum and energy fluxes. The new complete 
set of continuum equations can be tested against a variety of non-local-equilibrium gas flow problems, such as thermophoresis, shock waves and the Fourier heat transfer problem. These will be considered in the future work.

\section{ACKNOWLEDGMENTS}

Nishanth Dongari, Yonghao Zhang and Jason Reese would like to thank the European Community's Seventh Framework Programme FP7/2007-2013 for support through grant agreement ITN GASMEMS no. 215504.

\section{REFERENCES}

1. M. Gad-el-Hak, Journal of Fluids Engineering, 121, 5-33 (1999).

2. M. Knudsen, Annalen der Physik 28, 75-130 (1909).

3. F. Sharipov, Journal of Micromechanics and Microengineering, 9, 394-401 (1999).

4. T. Ewart, P. Perrier, I. A. Graur and J.G. Meolans, Journal of Fluid Mechanics, 584, 337-356 (2007).

5. H. C. Öttinger, Beyond Equilibrium Thermodynamics, Wiley, Hoboken, New Jersey, 2005.

6. H. Brenner, Navier-Stokes revisited, Physica A, 349, 60 (2005).

7. S. Chakraborty and F. Durst, Physics of Fluids 19, 088104, 1-4 (2007).

8. B. C. Eu, Journal of Chemical Physics, 128, 204507 (2008).

9. S. K. Dadzie, J. M. Reese and C. R. McInnes, Physica A 387, 6079-6094 (2008).

10. S. K. Dadzie, J. M. Reese, Physics of Fluids, 22, 016103 (2010).

11. N. Dongari, F. Durst and S. Chakraborty, Microfluidics and Nanofluidics, DOI:10.1007/s10404-010-0604-5 (2010).

12. H. C. Öttinger, H. Struchtrup and M. Liu, Physical Review E 80, 056303 (2009).

13. K. Pong, C. M. Ho, J. Liu, Y. C. Tai, Proceedings of Application of Microfabrication to Fluid Mechanics, ASME Winter Annual Meeting, Chicago, 51-56 (1994).

14. J. C. Maxwell, Philosophical Transactions of the Royal Society Part 1 170, 231-256 (1879).

15. N. Dongari, A. Agrawal and A. Agrawal, International Journal of Heat and Mass Transfer 50, 3411-3421 (2007).

16. T. Ohwada, Y. Sone, and K. Aoki, Physics of Fluids A 1, 2042-2049 (1989). 